in that disease. We also know that systemic actinomycosis may arise from alveolar infection due to the ray fungus.

Systemic disease due to a focus of infection anywhere, is probably always hematogenous. The study of the infected tissues of experimentally inoculated animals and the infected muscles, joint tissues, lymphnodes proximal to infected joints, nodes on tendons, e'c., of patients, yield specific bacteria, and histologically there is found embolism of the small and terminal blood-vessels. Local hemorrhage and endoarterial proliferation result in interstitial overgrowth, cartilaginous, osseous, vegetative and other morbid anatomical changes, dependent on the character of the tissue infected.

Partial or complete ischemia of the tissues due to the embolism is an important factor in the production of the morbid anatomic changes. Oxhausen of Berlin has produced, in animals, aseptic osteochondritis resembling arthritis deformans, by ligating the arteries supplying the joint tissues. These principles are, I think, susceptible of proof, that a chronic alveolar infection, and chronic foci in other regions also, may cause systemic disease by hematogenous bacterial emboli, which infect and at the same time deprive the tissues of nourishment. Local infection of muscles, joint tissues, etc., and lessened blood-supply result in the peculiar morbid anatomy of the respective tissues.

To investigate and manage these patients requires team work of the clinical and laboratory workers. The clinician must carefully examine the patient, exhausting every detail in the personal history. The skill of the dentist, the nose and throat specialist, the gynecologist, the genito-urinary expert and others may be necessary to locate the foci of infection. The focus must be destroyed. Tissues and exudates of foci should be carefully examined and bacterial cultures made. Vaccines of the dominant bacteria may be made for subsequent use.

With the source of the infection removed, the attempt should be made to increase the defenses of the body against the systemic infection. This involves a long and tedious period in the chronic joint infections and also in some other systemic diseases of focal origin. Rest, both mental and physical, is essential. Good, wholesome food, pure air, optimistic surroundings and, if necessary, restorative tonics of iron and other drugs. Pain must be palliated by simple drugs like the salicylates. After a time passive exercise and later active graduated exercise must be followed to aid in the restoration of local circulation and to hasten restoration of the morbid changes of the tissues. Autogenous vaccines may be used in the attempt to improve the defenses of the body. In chronic arthritis, with the circulation of the infected tissues obstructed embolically, antibodies in the blood stream, even if augmented by vaccines, would have but little effect locally. In the later stages of chronic arthritis when the local circulation has been so improved that the tissues are flooded with blood, vaccines will be of undoubted value. Patients may, however, be cured of chronic arthritis by the other measures named without the use of vaccines.

To recognize the character of this class of systemic disease and to manage successfully the patient, the physician must have absolute control. This implies hospital care where team work of the staff may insure quaîfied clinical and laboratory workers.

\section{MOUTH INFECTION AS A SOURCE OF SYSTEMIC DISEASE *}

\author{
C. H. MAYO, M.D.
}

ROCHESTER, MINN.

It has taken a long time for the general public to appreciate the full rôle of infection in the production of death, while even in the medical profession, more has come from the study of infections in the prevention of disease than in increasing the means of cure of disease, great as have been the results of treatment.

Since all animal life depends on"some other form of cell life, vegetable or animal, it seems but the part of all life to carry on this process of germinative development and maturity. It is only the resistance of healthy cells that prevents the inroads of the myriads of ever-present bacteria and animal parasites which are striving to get a foothold that they may in turn carry on their life work. Disease, then, is an inflammatory process from infection and the efforts at repair. It may also be chronic from the failure of cell life through lack of defense, from defective nutrition and advancing age.

We still speak more or less lightly of the so-called diseases of childhood, and the time is not far past when mothers took their children to be exposed to whooping cough, mumps, etc. To-day the intelligent woman knows that it is not necessary that the vitality of the child should be jeopardized by such preventable diseases, and that when they occur it is through carelessness, neglect or ignorance on the part of some one.

A comparatively small number of infections occur through wounds of the cutaneous surface. Many of those affecting the special organs are incurred through direct or indirect contact, as those of the eye and of the genito-urinary system. They may make most serious inroads on the general health. Infections which produce the greatest number of diseases enter the system by way of the alimentary and respiratory tracts. Somewhere in the line, then, of the alimentary and respiratory tracts and in the excretory ducts of the body lie the sources of the entrance of organisms which terminate life in the majority of instances. The great importance of the well-known diseases of the nasal passages with their sinuses, the lymphoid tissue of the pharynx, including the tonsils, and the diseases of gums and teeth, which have been given prominence by the dental profession during the last three years, is now more generally appreciated.

The mouth is the harbor of many varieties of bacteria which are constantly taken into the stomach during the process of eating. We have long looked on the acids of the stomach as destructive to such bacteria, but Smithies, in a microscopic examination of gastric extracts from 2,406 different individuals with "stomach complaint"' (dyspepsia, indigestion and the like), showed that irrespective of the degree of acidity of such gastric extracts, bacteria were present in 87 per cent. Morphologically cocci and diplococci were present in 83 per cent.; short and long rods (often of the colon group) in 58 per cent.; typical streptococci and staphylococci in 17 per cent. and Leptothrix buccalis in 24 per cent. In fifty-four cultural studies of saliva from "dyspeptic" patients, streptococci and staphylococci were demonstrated in over 80 per cent., bacilli in 66 per cent. and Leptothrix

* Address before the Section on Stomatology, American Medical Association, Atlantic City, June 24, 1914, in a symposium on Mouth Infection. 
buccalis in more than 14 per cent. Comparing these figures, it would appear that the common forms of pus-producing organisms (streptococci and staphylococci) have their proliferation retarded in gastric juice, but that bacilli (often of the colon group) as well as Leptothrix buccalis thrive in the stomach.

Bacteria of various forms live in the small intestine, or at least pass through it or into the blood stream by way of the mucous membrane. They exist in such great numbers in the large bowel that, whether living or dead, they constitute a considerable bulk of the dejecta. During the last few years some important points have been added to our knowledge of bacteria. Living germ life in the blood, or bacteremia, occurs in all infectious diseases. According to their number and virulence, the blood responds in slight or extreme degree to the symptoms, general and local, constituting the disease.

We have long known that bacteria were specified in type and action in all diseases in which we have been able to identify a specific germ. Rosenow has done a great work in showing that changes in environment may so change bacteria that specific action varies. The appearance of the bacteria is also unlike that of the original cell. In the blood stream these various forms, once they enter it, are selective in choosing their location, thereby developing specific local disease. The old "idiopathic" osteomyelitis of the child we now know may follow a short time after a specific tonsillitis. Pyorrhea, tonsillitis or sinus disease may be the source of an infection which we call rheumatism. Root abscesses and pus pockets connecting with them are often the source of acute and chronic rheumatism. The nasal sinuses and chronic mouth and throat infections develop anaphylaxis from the constant poisoning, and their results are shown in hay fevers, asthmas, urticarias, etc. Rosenow's work is going far to show that ulcerations of the stomach are conditions in which the mucosa is attacked from behind through the blood stream by bacteria which live in the blood and have a selective affinity for these particular areas. Septic bile which, in the majority of instances, is caused by infection is carried to the liver through the portal circulation. It creates such changes in the bile that it fails to activate the pancreatic and duodenal secretion, thus making various phases of indigestion, with qualitative rather than quantitative food trouble. Lower down we have the appendix with its lymphoid tissue which approximates in character that of the tonsil. Here the acid types of bacteria have the same opportunity, could they but enter the blood stream, of making erosions of the gastric mucosa as the specific form which is found in the mouth. We may here note that acid-secreting or acid-bathed surfaces are very subject to cancerous change, while alkaline-bathed surfaces are much less liable to be involved. Saliva is neutral or slightly alkaline in health, yet less than 20 per cent. of people have healthy mouths. The infected mouth shows a tendency to the acid reaction and it is through this acid change that we have an additional danger in cell degeneration, of malignant type, from chronic irritation.

The stomach is the most common location of all cancers, while the alkaline small intestine is rarely subject to cancer and the duodenum is most resistant to it. The large bowel again reverts to an acid reaction and is very subject to cancer. So also is the bladder. These structures with acid secretion are of more recent development than are many tissues of animal life, several of them being classed as organs of convenience, which fact renders them possibly less resistant.

Certainly enough is known concerning infections and their mode of entrance, that the infected and diseased mouth and respiratory tract must be looked on as most serious menaces. Much may be done by more general and effective school inspection. The present generation of children will understand and demand protection for their children in time. The first teeth should be watched, that the second be not permitted to erupt irregularly, causing deformities. Jaws should be spread that the teeth may meet and the high arched palate, diminishing nasal breathing, thereby reduced. Tonsils and adenoids should be looked after, thus preventing ear and mastoid diseases, rheumatism, endocarditis, etc. In chronic and recurring diseases, a search must be made to establish positively the nonparticipation of each of the several sources of infection.

The physicians engaged in this line of observation require fully as much training in the rudiments of dentistry as the dentist does in the signs of infectious diseases. While we have leaders in all professions, through the energy of their kinetic glands, the big stick which leads to our advancement is in the hands of the progressive and educated public who are constantly demanding more of their dentists, of the medical profession, and of the state in protecting them against preventable diseases.

\section{MOUTH INFECTION AS A SOURCE OF SYSTEMIC DISEASE *}

\section{E. C. ROSENOW, M.D. CHICAGO}

Cultures from emulsified tissues in various systemic disorders, such as the adjacent lymphatic glands draining the involved joints in arthritis deformans, in acute articular rheumatism, and the lymph-glands in Hodgkin's disease, have yielded interesting results. One striking thing in connection with some of the more chronic infections is that the character of the micro-organisms found in the lesion may be quite different from the character of the micro-organisms in the focus of infection at the same time. This, however, does not minimize the importance of the focus of infection in any way. The organisms found in the tissues may have undergone change. This fact should be borne in mind whenever autogenous vaccines are to be used. The poor results in some cases of arthritis, for example, following the use of autogenous vaccines prepared from the tonsils or other presumable focus, may be due to the fact that the organisms present in the focus at the particular time when the cultures were made were not like those actually infecting the tissues. And if so, the vaccine would fail to contain the proper antigen.

While the most common location of the focus in various infections is probably in the head, and hence the great opportunity for you, it may be located elsewhere. Thus in two cases of typical rheumatism we succeeded in isolating the Streptococcus rheumaticus from the stool; in one from an infected ingrowing

* Address before Section on Stomatology, American Medical Association, Atlantic City, June 24,1914 , in a symposium on Mouth Infection. 